\title{
Antiamnesic and Neuroprotective Effects of an Aqueous Extract of Ziziphus jujuba Mill. (Rhamnaceae) on Scopolamine-Induced Cognitive Impairments in Rats
}

\author{
Etienne Djeuzong, ${ }^{1}$ Antoine K. Kandeda ${ }^{(D},{ }^{2}$ Séfirin Djiogue, ${ }^{2}$ Lewale Stéphanie, ${ }^{1}$ \\ Danide Nguedia, ${ }^{1}$ Florence Ngueguim, ${ }^{2}$ Jean P. Djientcheu, ${ }^{2}$ Jonas Kouamouo, ${ }^{1}$ \\ and Théophile Dimo (iD) ${ }^{2}$
}

${ }^{1}$ Department of Pharmacy, University of Montagnes, P.O. Box 208, Banganté, Cameroon

${ }^{2}$ Department of Animal Biology and Physiology, University of Yaoundé I, P.O. Box 812, Yaoundé, Cameroon

Correspondence should be addressed to Antoine K. Kandeda; kandedakavaye@yahoo.fr and Théophile Dimo; dimo59@yahoo.com

Received 20 January 2021; Revised 13 May 2021; Accepted 2 August 2021; Published 11 August 2021

Academic Editor: Alin Ciobica

Copyright (c) 2021 Etienne Djeuzong et al. This is an open access article distributed under the Creative Commons Attribution License, which permits unrestricted use, distribution, and reproduction in any medium, provided the original work is properly cited.

\begin{abstract}
Background. Alzheimer's disease is a neurological condition that affects about 44 million people worldwide. The available treatments target symptoms rather than the underlying causes. Ziziphus jujuba (Rhamnaceae) is widely used in traditional Cameroonian medicine to treat diabetes, pain, infections, and dementia. Previous studies reported that $Z$. jujuba aqueous macerate improves working memory impairment, but no study on the antiamnesic effect of a concoction of $Z$. jujuba in rats has been performed. Therefore, this study aimed to assess the antiamnesic and neuroprotective effects of an aqueous extract of Z. jujuba on scopolamine-induced cognitive impairments in rats. Methods. Learning and memory impairments were induced in rats by administering scopolamine ( $1 \mathrm{mg} / \mathrm{kg}$, i.p.) to 58 rats for 15 days. Rats that developed learning and memory impairments in Morris water maze and Y-maze paradigms were divided into 7 groups ( 8 rats each) and treated daily for 15 days as follows: the normal control group received distilled water $(10 \mathrm{ml} / \mathrm{kg}$, p.o.), the negative control group received distilled water (10 $\mathrm{ml} / \mathrm{kg}$, p.o.), positive control groups either received donepezil $(1.2 \mathrm{mg} / \mathrm{kg}$, p.o.) or tacrine $(10 \mathrm{mg} / \mathrm{kg}$, p.o.), and the three test groups were given the extract $(29,57$, and $114 \mathrm{mg} / \mathrm{kg}$, p.o. $)$. At the end of treatments, learning and memory impairments were determined using the same paradigms. Animals were then euthanized, and biochemical parameters of oxidative stress, inflammation, and apoptosis were analyzed in the hippocampus and prefrontal cortex. Results. On the 4th day of the acquisition phase in the Morris water maze, $Z$. jujuba (29 and $114 \mathrm{mg} / \mathrm{kg})$ reduced $(p<0.001)$ the latency to reach the platform, while in the retention phase, $Z$. jujuba (57 and $114 \mathrm{mg} / \mathrm{kg})$ decreased $(p<0.001)$ the time to reach the platform and increased the time in the target quadrant $(p<0.05)$ compared to control. Surprisingly, the extract failed to affect spontaneous alternations in the $Y$-maze. Furthermore, the extract $(29,57$, and $114 \mathrm{mg} / \mathrm{kg})$ reversed $(p<0.001)$ scopolamine-induced oxidative stress, inflammation, and apoptosis. This was supported by the reduction of neuronal alterations in the hippocampus and prefrontal cortex. Conclusions. Compared to donepezil, a standard drug against Alzheimer's disease, these findings suggest that Z. jujuba extract possesses antiamnesic and neuroprotective effects, and these effects are mediated in part through antioxidant, anti-inflammatory, and antiapoptotic activities. These findings help to explain its use in treating psychiatric disorders in Cameroon's folk medicine.
\end{abstract}

\section{Introduction}

Alzheimer's disease (AD) is the most common form of dementia (60-70\% of cases) [1]. It is an irreversible and progressive neurodegenerative disorder of the central nervous system that occurs gradually and leads to memory loss, unusual behavior, and personality changes $[1,2]$. According to the World Health Organization (WHO), more than 44 million people worldwide are affected by $\mathrm{AD}$, with 7.7 million new cases annually [3-5]. In Africa, the 
prevalence of $\mathrm{AD}$ is estimated at $5.6 \%$, with $0.96 \%$ in Cameroon [6]. At the molecular and cellular levels, $\mathrm{AD}$ is characterized by extracellular deposits of beta-4-amyloid protein (BAP), intracellular tangles, cholinergic deficit, extensive neuronal loss, and synaptic changes in the cerebral cortex and hippocampus [7]. Of all types of dysfunctions, BAP plaques and neurofibrillary tangles are the main cytologic hallmarks. BAP seems to initiate the pathogenesis of $\mathrm{AD}$, while neurofibrillary tangles seem to be mainly involved in its progression $[2,3]$. BAP deposits are associated with neuronal death via oxidative stress, inflammation, and apoptosis [2]. AD can be modeled in rodents using chemicals, such as D-galactose, glutamate, and scopolamine. Scopolamine, a muscarinic cholinergic receptor antagonist, is used as a pharmacological experimental model for inducing cognitive impairments and memory disorders in animals. Indeed, cognitive behaviors, such as ataxia, anxiety, depression, short-term memory, reference memory, and attention are affected by injections of scopolamine in rodents [1]. Both the hippocampus and prefrontal cortex are susceptible to oxidative stress, and excessive oxidative stress induces memory deficiency by damaging synaptic plasticity and causing inflammation and neuronal cell death [2]. It was found that when scopolamine is injected in rodents, it induces oxidative stress, neuronal inflammation, and apoptosis in the brain [2].

At present, there is no curative treatment for $\mathrm{AD}$ [8]. Drug therapies are available, including acetylcholinesterase inhibitors (galantamine, rivastigmine, and donepezil) and the N-methyl-D-aspartic acid receptor antagonist (memantine) [9]. However, these treatments are costly, difficult to access, have significant side effects, and all only relieve symptoms and do not treat the underlying causes $[2,3]$. Therefore, there is a need to develop an effective treatment against $\mathrm{AD}$.

Medicinal plants, particularly those used in traditional herbal medicine, constitute a source for discovering new candidates for effective and safe drugs for AD. Among them, Ziziphus jujuba, a plant from the Rhamnaceae family, is used in Cameroon, Asia, and India to treat multiple pathologies, including typhoid fever, furuncle, sleep disorders, diarrhea, and pain $[10,11]$. In Northern Cameroon, according to claims of traditional healers, all parts of the plant are used to treat otitis, inflammation, cancer, anxiety, rickets, typhoid fever, and anorexia; the seeds are used as dewormers [12] and the leaves for treating cases of dementia [12]. In recent years, research on $Z$. jujuba fruits, seeds, and leaves has revealed anti-inflammatory [13] and neuroprotective activities [14]. The leaves exhibited anti-inflammatory [15], antifungal, anticancer, antifertility, antibacterial, anxiolytic, sedative, and antioxidant properties [16-18]. Pharmacological studies on the antiamnesic effect of $Z$. jujuba reported that the seed has a protective effect on spatial memory impairments in rats [19-21]. According to these authors, this effect was likely mediated by cholinergic blockade [22]. In addition, a hydroethanolic extract of $Z$. jujuba has been shown to ameliorate cognitive decline and seizures in an experimental model of epilepsy in rats [20]. A toxicity study on the leaves of $Z$. jujuba revealed no significant toxicities
[23], though toxic elements are found in trace amounts in the whole plant [24]. Phytochemical analysis of the seeds, leaves, and stem bark of $Z$. jujuba indicated alkaloids, flavonoids, tannins, saponins, and polyphenols [25]. Moreover, the HPLC fingerprint of $Z$. jujuba leaf extracts identified the presence of major specific constituents including (-)-catechin, traumatic acid, quercetin-3-O-robinobioside, rutin, and quercetin-3-O- $\alpha$-L-arabinosyl- $(1 \longrightarrow 2)-\alpha$-L-rhamnoside, with a total of nine flavonoids identified [26]. Furthermore, GC/MS analysis of the ethanol extract of $Z$. jujuba seeds revealed the existence of 20 components, mainly 13heptadecyn-1-ol (12.95\%), 7-ethyl-4-decen-6-one (9.73\%), lineoleoyl chloride (8.54\%), linoleic acid (6.37\%), 2,5-octadecadiynoic acid, methyl ester (5.57\%), and palatinol A (4.81\%) [27]. Numerous specific chemicals present in the extract have been identified, some of which have been demonstrated to possess anti-inflammatory, antioxidant, and antimalarial activities [26].

To date, there is no solid evidence for the antiamnesic and neuroprotective effects of extracts from Z. jujuba leaves, one of the most promising therapeutic properties of the extract. Therefore, this study was undertaken to investigate the antiamnesic and neuroprotective effects of $Z$. jujuba aqueous extract on scopolamine-induced cognitive impairments in rats, using Morris water maze and Y-maze paradigms. Besides, biochemical assays for antioxidant, antiinflammatory, and antiapoptotic effects and their mechanisms were explored.

\section{Materials and Methods}

2.1. Plant Collection and Extraction. The leaves of Z. jujuba were harvested in Mokolo (far north region of Cameroon) at global position system coordinates $10.7425^{\circ}$ north and $13.8042^{\circ}$ east and identified at the National Herbarium of Cameroon (HNC) (database of herbarium index: http:// sweetgum.nybg.org/science/ih/herbarium-list) by $\mathrm{Mr}$. Ngansop Eric in comparison to the specimen deposited under the voucher number $14446 / \mathrm{HNC} / \mathrm{Cam}$. The plant name has also been checked on http://www.theplantlist.org. The extract of $Z$. jujuba was prepared according to the traditional healers' method. Briefly, fresh leaves of Z. jujuba were dried in the shade and crushed into powder. The powder $(75 \mathrm{~g})$ was then boiled in 1.51 of distilled water for $20 \mathrm{~min}$, giving a filtered mixture. The filtrate was finally dried at $50^{\circ} \mathrm{C}$ to a dry extract $(10.8 \mathrm{~g})$, yielding $14.4 \%(w / w)$. Stock solutions of $2.9 \mathrm{mg} / \mathrm{ml}, 5.7 \mathrm{mg} / \mathrm{ml}$, and $11.4 \mathrm{mg} / \mathrm{ml}$ concentrations were prepared for administration to animals.

2.2. Drugs and Chemicals. Donepezil tablets were purchased from U.C.B. Pharma SA Braine-l'Alleud (Belgium) and tacrine hydrochloride capsules from Shionogi. Inc. (Japan). Scopolamine hydrobromide, trichloroacetic acid, ketamine, thiobarbituric acid, Ellman reagent, Griess reagent, adrenaline, and formalin were purchased from Sigma Chemical Co., St. Louis (United States), while diazepam was purchased from Roche (Switzerland). Donepezil, tacrine, and scopolamine were dissolved in distilled water. All solutions were 
administered per os (p.o.) at a volume of $10 \mathrm{ml} / \mathrm{kg}$ body weight, except for scopolamine, ketamine, and diazepam, administered intraperitoneally (i.p.).

2.3. Animals and Housing Conditions. Animals were male Wistar rats, 6 to 8 weeks old, weighing between 120 and $140 \mathrm{~g}$. Rats were raised in the animal facilities of the Laboratory of Animal Physiology (University of Yaoundé I, Cameroon) under standard light (12-hour day/night cycle), temperature $\left(24 \pm 2^{\circ} \mathrm{C}\right)$, and humidity $\left(45 \pm 3^{\circ} \mathrm{C}\right)$ with access to standard animal diet and tap water ad libitum. The animals were housed in groups of 5 rats per cage $(40 \mathrm{~cm} \times 40 \mathrm{~cm})$. Animal procedures were carried out following the guidelines of the Institutional Ethics Committee of the Cameroon Ministry of Scientific Research and Technological Innovation (Reg. no. FWA-IRD 0001954, 04/ 09/2006), which adopted the guidelines of the European Union on Animal Care (C.E.E. Council 86/609). Euthanasia of animals was performed according to the American Veterinary Medical Association (AVMA) guidelines for the euthanasia of animals (2020). All animal studies, including allocating animals to experimental groups, experimental dosages, outcomes, and statistical methods, are detailed and carried out according to ARRIVE guidelines 2.0 (http:// www.nc3rs.org.uk/page.asp?id=1357). For behavioral experiments, animals were randomly selected.

2.4. Experimental Design and Treatment. To induce learning and memory impairments, 58 rats were randomly divided into two groups and treated for 15 days as follows:

(i) A scopolamine group of 50 rats was treated with scopolamine ( $1 \mathrm{mg} / \mathrm{kg}$, i.p.)

(ii) A normal control group of 8 rats was given distilled water $(10 \mathrm{ml} / \mathrm{kg}$, i.p.)

At the end of the treatments, learning and memory impairments were assessed with the Morris water maze and Y-maze paradigms. Rats that developed learning and memory impairments were selected for further studies. These rats were divided into 7 groups of 8 rats each and treated as follows:

(i) A negative control group that received distilled water $(10 \mathrm{mg} / \mathrm{kg}$, p.o.)

(ii) Two positive control groups that received donepezil $(1.2 \mathrm{mg} / \mathrm{kg}$, p.o.) or tacrine $(10 \mathrm{mg} / \mathrm{kg}$, p.o.)

(iii) Three test groups that received the aqueous extract of $Z$. jujuba at the doses of 29,57, and $114 \mathrm{mg} / \mathrm{kg}$, respectively, p.o.

A normal control group was added as the $7^{\text {th }}$ group $(n=7)$ and treated with distilled water $(10 \mathrm{ml} / \mathrm{kg}$, p.o.). All rats were administered the test agents for 15 additional days, and then, cognitive decline or its improvement was assessed with the same water maze and Y-maze paradigms. After completion of the behavioral procedures, rats were sacrificed and brains were removed for analysis of biochemical markers $(n=5)$ and histological assays $(n=2)$ (Figure 1$)$.

\subsection{Behavioural Studies}

2.5.1. Morris Water Maze Test. Spatial learning and longterm memory impairments were assessed using the Morris water maze test [3] with a circular tank of $150 \mathrm{~cm}$ diameter and $60 \mathrm{~cm}$ height filled to $40 \mathrm{~cm}$ with water at $25^{\circ} \mathrm{C}$. The tank was divided into four equal quadrants with a white refuge platform of $8 \mathrm{~cm}$ diameter and $30 \mathrm{~cm}$ height placed in the center of one of the quadrants $1 \mathrm{~cm}$ below the water surface. The pool was located in a room with multiple visual cues. On the 1st day of the test, i.e., during the habituation phase, each rat was acclimated for $60 \mathrm{~s}$ without the platform. Each rat accomplished three-block sessions with intervals of $30 \mathrm{~min}$ between sessions; each block consisted of four successive trials with $60 \mathrm{~s}$ duration. On each trial, rats were randomly released into the water from one of the four quadrants facing the maze wall $[4,5]$. The acquisition phase started on the $2^{\text {nd }}$ day with the refuge platform and continued for 4 days with three sessions per day. The water was clouded by adding liquid milk so that the platform was invisible from the water surface. The session time for each animal to find the platform was $120 \mathrm{~s}$, and the time interval between sessions was $5 \mathrm{~min}$. When an animal found the platform, it was allowed to stay for $15 \mathrm{~s}$. During each session of the acquisition phase, the latency time to find the platform was recorded for each animal. The effectiveness of learning was then assessed in the retention phase on the $6^{\text {th }}$ day. During this phase, which lasted $120 \mathrm{~s}$, the platform was removed from the tank. Thus, the latency time to find the proper quadrant where the platform was previously and the time spent in this compartment were recorded [6].

2.5.2. Y-Maze Test. The Y-maze test was used to assess working memory in animals by recording spontaneous alternations [7]. The maze used was a wooden device with three identical branches $(40 \mathrm{~cm}$ long $\times 35 \mathrm{~cm}$ high $\times 12 \mathrm{~cm}$ wide) separated by an angle of $120^{\circ}$. The walls of each arm were decorated with different patterns to differentiate them and labeled as A, B, and C. Rats were individually placed at the end of a maze's branch for free exploration [8]. During $5 \mathrm{~min}$, the number of entries in each arm of the maze was recorded. After each session, the device was cleaned with $10 \%$ ethanol to avoid odors. A spontaneous alternation was defined as three successive entries in three different arms, for example, $\mathrm{ABC}, \mathrm{CAB}$, or BCA. The percentage of SA was used as an index of working memory and calculated according to the following formula: [(number of SA)/(total number of arms visited -2$)]^{*} 100$.

\subsection{Biochemical Analysis}

2.6.1. Animal Euthanasia and Preparation of Homogenates. Following the behavioral assessments, animals were immediately euthanized under anesthesia with ketamine (70 mg/kg, i.p.) and diazepam $(10 \mathrm{mg} / \mathrm{kg}$, i.p.). The brain of each rat was removed and divided into two hemispheres. The hippocampus and prefrontal cortex were isolated from one hemisphere, washed in $0.9 \% \mathrm{NaCl}$, and blotted to dryness. 


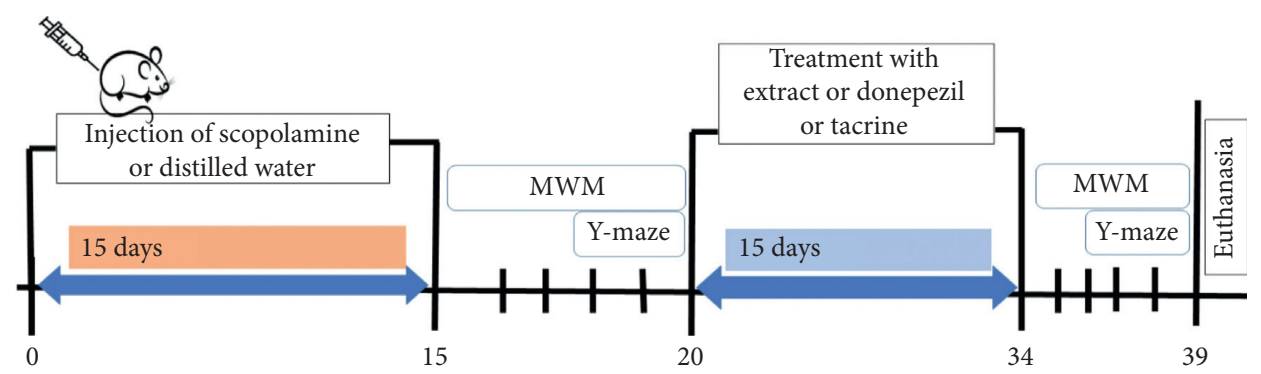

FIgURE 1: Schematic diagram of the experimental procedure. MWM: Morris water maze.

They were then weighed and homogenized in Tris- $\mathrm{HCl}$ buffer $(50 \mathrm{mM}, \mathrm{pH} 7.4)$ at a ratio of $20 \%(w / v)$. After centrifugation at $3000 \times \mathrm{g}$ at $4^{\circ} \mathrm{C}$ for $25 \mathrm{~min}$, the supernatant was collected into tubes and stored at $-20^{\circ} \mathrm{C}$ for further neurochemical evaluation. The other hemisphere of the brain was fixed in $4 \%$ formalin for later histological analysis.

2.6.2. Reduced Tissue Glutathione Concentration. Ellman reagent $(1.5 \mathrm{ml})$ was added to tubes containing $100 \mu \mathrm{l}$ of homogenate or Tris-buffer $(50 \mathrm{mM} \mathrm{HCl}, 150 \mathrm{mM} \mathrm{KCl}, \mathrm{pH}$ 7.4) as control. The tubes were shaken for $60 \mathrm{~min}$ at room temperature $\left(25 \pm 1^{\circ} \mathrm{C}\right)$, and then, absorbance at $412 \mathrm{~nm}$ was read against the control. The concentration of reduced glutathione (GSH) was expressed in $\mu \mathrm{mol} / \mathrm{g}$ of tissue proteins [9].

2.6.3. Malondialdehyde Concentration. The malondialdehyde (MDA) assay was carried out according to the method described by Wilbur et al. [10]. Briefly, $250 \mu$ l of homogenate was added to the test tubes and $250 \mu \mathrm{l}$ of Tris-buffer $(50 \mathrm{mM}$ $\mathrm{HCl} ; 150 \mathrm{mM} \mathrm{KCl} ; \mathrm{pH} 7.4)$ to the blank tube. Then, $125 \mu \mathrm{l}$ of $20 \%$ trichloroacetic acid and $250 \mu \mathrm{l}$ of $0.67 \%$ thiobarbituric acid were added to each tube, and the tubes were incubated for $10 \mathrm{~min}$ at $90^{\circ} \mathrm{C}$. They were then cooled and centrifuged at $3000 \times \mathrm{g}$ for $15 \mathrm{~min}$ at room temperature $\left(25 \pm 1^{\circ} \mathrm{C}\right)$. The supernatant was removed, and the absorbance at $530 \mathrm{~nm}$ was read against the blank. The concentration of MDA was expressed in $\mathrm{mmol} / \mathrm{g}$ of tissue protein.

2.6.4. Superoxide Dismutase Activity. The activity of SOD was determined according to the method of Misra and Fridovish [11]. In the blank tube, $1666 \mu \mathrm{l}$ of carbonate buffer ( $50 \mathrm{mM}, \mathrm{pH} 10.2$ ) was added, and $134 \mu \mathrm{l}$ of homogenate was added to each sample tube. The reaction was started by adding $200 \mu \mathrm{l}$ of $0.3 \mathrm{mM}$ adrenaline solution. After fast inversion for mixing, the absorbance at $480 \mathrm{~nm}$ was read after 20 and $80 \mathrm{~s}$. The specific activity of SOD was expressed in units of $\mathrm{SOD} / \mathrm{min} / \mathrm{g}$ of tissue protein.

2.6.5. Nitrite Concentration. According to the method of Grand et al. [12], the determination of nitrite concentration was carried out. Nitric oxide is a short-half-life compound that is rapidly converted to stable end products, which comprise nitrate (NO3-) and nitrite (NO2-). The conversion of nitrate into nitrite is followed by color development in the presence of $0.1 \% \mathrm{~N}$-(1-naphthyl) ethylenediamine dihydrochloride, $1 \%$ sulfanilamide, and $2.5 \%$ phosphoric acid in an acid medium (Griess reagent). Briefly, $200 \mu \mathrm{l}$ of homogenate and $200 \mu \mathrm{l}$ of Griess reagent were introduced into test tubes. The solutions were then mixed, and the absorbance was read at $570 \mathrm{~nm}$ after $10 \mathrm{~min}$ against the blank. Furthermore, a standard curve was established with a set of serial dilutions of nitrite. The obtained equation was used to calculate the unknown sample concentration. The nitrite concentration in the homogenate was expressed in $\mathrm{mmol} / \mathrm{g}$ of protein in the tissue [13].

2.6.6. Total Protein Concentration. Total protein concentration in the extract was carried out according to the method of Gornall et al. [14], with the protein concentration expressed in $\mu \mathrm{g} / \mathrm{ml}$.

2.6.7. Proinflammatory Markers Concentration. The concentrations of TNF- $\alpha$, IL1- $\beta$, and IL- 6 were determined by Enzyme-Linked Immunosorbent Assay (ELISA) using the Quantikine kit ( $\mathrm{R}$ and D Systems, Inc., Minneapolis, USA). The concentration of these cytokines was expressed in $\mathrm{pg} / \mathrm{ml}$.

2.6.8. Apoptosis Marker Concentration. Quantification of caspases 3 and 9 was carried out by ELISA using the Novus Biologicals kit (R and D Systems, Inc., Minneapolis, USA). The concentration of these apoptosis markers was expressed in $\mathrm{ng} / \mathrm{ml}$.

2.7. Histopathological Analysis of Brain Tissues. The histological analysis included fixing, cutting, dehydration, inclusion, cutting, coloring, mounting, and observation. The stained and mounted slides were observed at 250X magnification using a Scientico STM-50 optical microscope (HSIDC Industrial Estate, Haryana, India) equipped with a Celestron 44421 digital camera connected to a computer (HP Pavilion, g series). Furthermore, the density of neurons in CA1 and CA3 layers of the hippocampus was determined by counting the number of neurons per $400 \mu \mathrm{m}^{2}$.

2.8. Qualitative Phytochemical Screening. The method of Odebiyi and Sofowora [15] was used to screen flavonoids, alkaloids, saponins, tannins, anthraquinones, triterpenes, and polyphenols, while reducing sugars were ascertained 
using the method of Harbone [16]. The following reagents were used: flavonoids $(\mathrm{NaOH}$ and $\mathrm{HCl})$, alkaloids $\left(\mathrm{H}_{2} \mathrm{SO}_{4}{ }^{-}\right.$and Meyer's reagent), saponins (DMSO, frothing test), polyphenols (DMSO, K3F (CN)6), tannins (DMSO, ferric chloride), reducing sugar (Lieberman's test), and triterpenes ( $\mathrm{H}_{2} \mathrm{SO}_{4}^{-}$and acetic anhydride).

2.9. Quantitative Phytochemical Screening. The concentrations of total phenols, total flavonoids, condensed tannins, total alkaloids, and saponins were determined as previously described by Hatami [17], Dehpour et al. [18], Ba [19], Gracelin [20], and Gracelin [20], respectively.

2.10. Statistics. Statistical analyses of the values obtained were carried out using GraphPad Prism version 7.1. (San Diego, CA, USA). Results were expressed as the mean\pm standard error of the mean (SEM). Data analyzed were first assessed for normality and sphericity using Shapiro-Wilk and Mauchly's tests, respectively. These tests confirmed that data were normally distributed, but some of them violated the assumption of sphericity. Therefore, the Greenhouse-Geisser test was used for correction. Repeated one-way ANOVA followed by Tukey's post hoc test was used to analyze data from the number of spontaneous alternations in the Y-maze test and oxidative stress, proinflammatory cytokines, and apoptosis markers. Repeated two-way ANOVA followed by the Bonferroni post hoc test was used to analyze data from the Morris water maze test. At $p<0.05$, the difference was considered significant.

\section{Results}

3.1. Effect of Z. jujuba Extract on the Acquisition Phase in the Morris Water Maze. The effect of the extract of $Z$. jujuba during the acquisition phase is shown in Table 1 . On the 4 th day of the acquisition phase, scopolamine significantly $(p<0.001)$ induced learning deficits in the negative control group when compared to the normal control group ( $\mathrm{Ta}$ ble 1). In contrast, the extract of Z. jujuba (29 and $114 \mathrm{mg} /$ $\mathrm{kg})$ showed the highest $(p<0.001)$ decrease in the latency to reach the platform (Table 1 ). The effect of the extract was of similar magnitude to that of donepezil, a standard drug against $\mathrm{AD}$ known to have this effect (Table 1).

\subsection{Effect of Z. jujuba Extract on the Retention Phase in the} Morris Water Maze. The effect of the extract of Z. jujuba during the retention phase is shown in Figure 2. During this retention phase, administration of scopolamine in the negative control group significantly $(p<0.001)$ increased the latency to reach the platform when compared to the normal control group (Figure 2(a)). However, the extract of $Z$. jujuba significantly $(p<0.001)$ decreased the latency to reach the platform at all concentrations tested. The effect of the extract was similar to that of donepezil, whereas the decrease by tacrine was not significant (Figure 2(a)).

Also, during this phase, administration of scopolamine in the negative control group significantly $(p<0.001)$ decreased the time spent in the target quadrant when compared to the normal control group (Figure 2(b)). In contrast, the extract at the dose of $57 \mathrm{mg} / \mathrm{kg}$ showed the highest $(p<0.01)$ increase in the time spent in the target quadrant $(40.9 \pm 2.0 \mathrm{~s})$ (Figure 2(b)). This effect of the extract was comparable to that of donepezil, which increased this time to $38.7 \pm 1.9 \mathrm{~s}(p<0.05)$ (Figure 2(b)).

Each bar represents the average \pm SEM; $n=7 .{ }^{c} p<0.001$ vs. normal control. ${ }^{*} p<0.05$; ${ }^{* *} p<0.01$; $^{* * *} p<0.001 v s$. negative control. DW: distilled water $(10 \mathrm{ml} / \mathrm{kg})$; SCO: scopolamine (1 mg/kg); DZ: donepezil $(1.2 \mathrm{mg} / \mathrm{kg})$; TA: tacrine $(10 \mathrm{mg} / \mathrm{kg})$; and E29, E57, and E114: aqueous extract of $Z$. jujuba at respective doses of 29,57 , and $114 \mathrm{mg} / \mathrm{kg}$. $\mathrm{DW}+\mathrm{DW}$ : normal control group; $\mathrm{SCO}+\mathrm{DW}$ : negative control group; $\mathrm{SCO}+\mathrm{DZ}$ : positive control group treated with donepezil; SCO + TA: positive control group with tacrine; and SCO+E29-E114: test groups treated with Z. jujuba extract.

\subsection{Effect of Z.jujuba Extract on the Spontaneous Alternations} in the Y-Maze. The Y-maze test results are presented in Figure 3. A significant $(p<0.01)$ reduction was observed in the number of spontaneous alternations between the negative control group and the normal control group. The extract at all doses, as well as donepezil or tacrine, showed a nonsignificant $(p>0.05)$ increase in this parameter (Figure 3).

Each bar represents the average \pm SEM; $n=7 .{ }^{\mathrm{b}} p<0.01$ vs. normal control. DW: distilled water $(10 \mathrm{ml} / \mathrm{kg})$; SCO: scopolamine (1 mg/kg); DZ: donepezil (1.2 mg/kg); TA: tacrine $(10 \mathrm{mg} / \mathrm{kg})$; and E29, E57, and E114: aqueous extract of $Z$. jujuba at respective doses of 29,57 , and $114 \mathrm{mg} / \mathrm{kg}$. $\mathrm{DW}+\mathrm{DW}$ : normal control group; $\mathrm{SCO}+\mathrm{DW}$ : negative control group; $\mathrm{SCO}+\mathrm{DZ}$ : positive control group treated with donepezil; SCO + TA: positive control group treated with tacrine; and SCO + E29-E114: test groups treated with Z. jujuba extract.

3.4. Effect of Z. jujuba Extract on Some Oxidative Stress Markers in the Hippocampus and Prefrontal Cortex. The effect of the extract of $Z$. jujuba on some oxidative stress markers in the hippocampus and prefrontal cortex is shown in Tables 2 and 3. In the hippocampus and prefrontal cortex, there was a significant $(p<0.001)$ decrease in the concentration of GSH between the negative control group and the normal control group (Tables 2 and 3). However, in the hippocampus, the extract at doses of 29 and $114 \mathrm{mg} / \mathrm{kg}$, as well as tacrine, showed a moderate $(p<0.05)$ increase in the concentration of GSH $(77.59 \%, 72.04 \%$, and $127.62 \%$, respectively), while in the prefrontal cortex, the extract $(29 \mathrm{mg} /$ $\mathrm{kg})$ and tacrine showed a significant $(p<0.001)$ increase in the concentration of GSH $(264.57 \%$ and $209.91 \%$, respectively) (Tables 2 and 3). Tables 2 and 3 show that, in the hippocampus and prefrontal cortex, there was a significant $(p<0.001)$ increase in the concentration of MDA between the negative control group and the normal control group (Tables 2 and 3). In contrast, the extract at all doses, donepezil, and tacrine significantly $(p<0.001)$ decreased the 
TABLE 1: Effect Z. jujuba extract on the latency to reach the platform during the acquisition phase in the Morris water maze.

\begin{tabular}{|c|c|c|c|c|}
\hline Treatment & Day 1 lat. (s) & Day 2 lat. (s) & Day 3 lat. (s) & Day 4 lat. (s) \\
\hline $\mathrm{DW}+\mathrm{DW}$ & $21.3 \pm 4.4$ & $8.3 \pm 0.8$ & $6.9 \pm 0.5$ & $5.9 \pm 0.2$ \\
\hline $\mathrm{SCO}+\mathrm{DW}$ & $29.3 \pm 3.5$ & $15.0 \pm 2.0$ & $14.1 \pm 2.2$ & $14.5 \pm 2.7^{\mathrm{C}}$ \\
\hline $\mathrm{SCO}+\mathrm{DZ}$ & $24.7 \pm 2.2$ & $13.3 \pm 2.6$ & $12.8 \pm 2.5$ & $6.1 \pm 1.1^{* *}$ \\
\hline $\mathrm{SCO}+\mathrm{TA}$ & $51.7 \pm 4.3$ & $10.6 \pm 1.8$ & $9.8 \pm 1.5$ & $10.9 \pm 1.4$ \\
\hline $\mathrm{SCO}+\mathrm{E} 29$ & $38.8 \pm 2.1$ & $9.8 \pm 0.9$ & $6.2 \pm 0.8$ & $5.2 \pm 0.4^{* * *}$ \\
\hline $\mathrm{SCO}+\mathrm{E} 57$ & $36.9 \pm 6.7$ & $6.9 \pm 0.7$ & $8.8 \pm 0.9$ & $7.2 \pm 0.7^{* *}$ \\
\hline $\mathrm{SCO}+\mathrm{E} 114$ & $27.2 \pm 3.4$ & $6.7 \pm 1.5$ & $7.4 \pm 0.6$ & $6.0 \pm 0.7^{* * *}$ \\
\hline
\end{tabular}

Each value represents the average $\pm \mathrm{SEM} ; n=7 .{ }^{\mathrm{c}} p<0.001$ vs. normal control. ${ }^{* *} p<0.01 ;{ }^{* * *} p<0.001$ vs. negative control. s: second; lat.: latency time; DW: distilled water (10 ml/kg); SCO: scopolamine (1 mg/kg); DZ: donepezil $(1.2 \mathrm{mg} / \mathrm{kg}) ;$ TA: tacrine $(10 \mathrm{mg} / \mathrm{kg}) ;$ E29, E57, and E114: aqueous extracts of Z. jujuba at respective doses of 29,57 , and $114 \mathrm{mg} / \mathrm{kg}$. DW + DW: normal control group; SCO + DW: negative control group; SCO + DZ: positive control group with donepezil; SCO + TA: positive control group treated with tacrine; SCO + E29-E114: test groups treated with Z. jujuba extract.

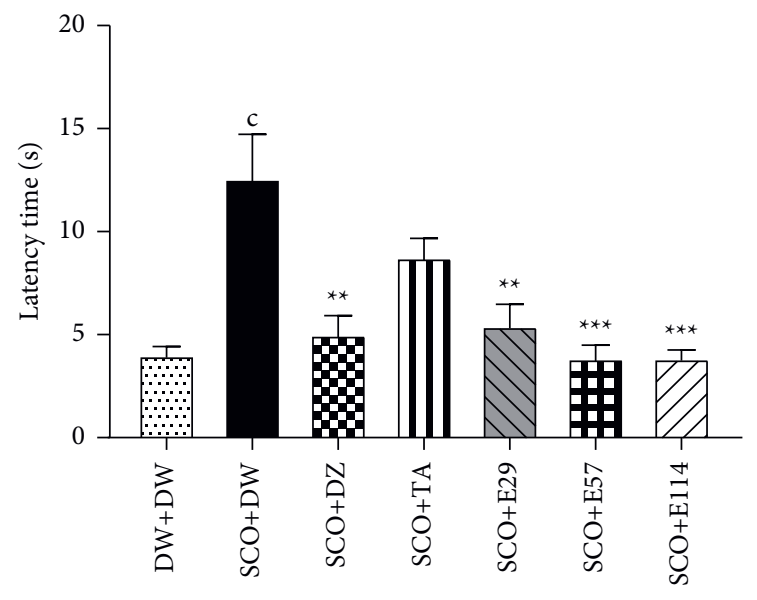

(a)

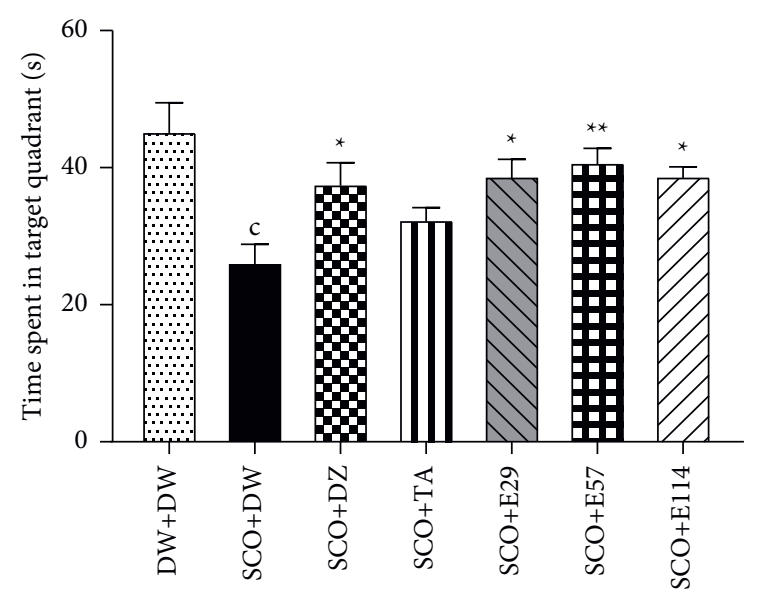

(b)

FIgURE 2: Effect Z. jujuba extract on the latency time to reach the platform (a) and the time spent in the target quadrant (b) during the retention phase in the Morris water maze.

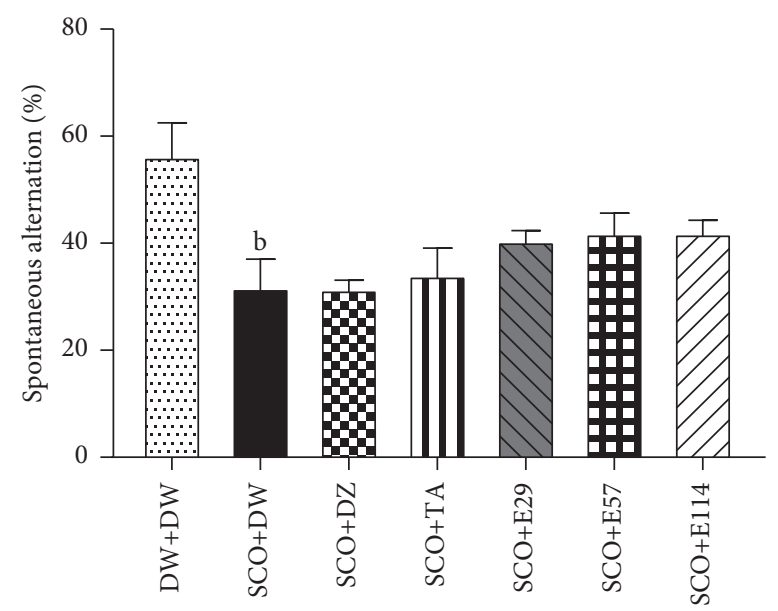

Figure 3: Effect of $Z$. jujuba extract on the percentage of spontaneous alternation in the Y-maze.

MDA concentration in the hippocampus, whereas in the prefrontal cortex, the extract $(114 \mathrm{mg} / \mathrm{kg})$ showed the highest $(p<0.01)$ reduction in this parameter (Tables 2 and 3$)$. The effect of the extract was similar to that of donepezil $(p<0.05)$ or tacrine $(p<0.01)$ (Tables 2 and 3 ).
In the hippocampus and prefrontal cortex, the activity of SOD significantly $(p<0.001)$ increased between the negative control group and the normal control group (Tables 2 and 3 ). The extract at all doses, as well as donepezil or tacrine, significantly $(p<0.001)$ reduced this 
TABLE 2: Effect of $Z$. jujuba extract on some oxidative stress markers in the hippocampus.

\begin{tabular}{lccccc}
\hline \multirow{2}{*}{ Treatment } & & \multicolumn{3}{c}{ Oxidative stress markers in the hippocampus } \\
& GSH $(\mu \mathrm{mol} / \mathrm{g})$ & MDA $(\mathrm{mmol} / \mathrm{g})$ & SOD $($ unit/min/g) & Nitrite $(\mathrm{mmol} / \mathrm{g})$ & Total protein $(\mu \mathrm{g} / \mathrm{ml})$ \\
\hline DW + DW & $690.1 \pm 48.3$ & $17.1 \pm 1.2$ & $14.4 \pm 0.8$ & $144.1 \pm 1.7$ & $1751.3 \pm 24.1$ \\
SCO + DW & $316.4 \pm 18.9^{\mathrm{c}}$ & $64.6 \pm 3.4^{\mathrm{c}}$ & $146.1 \pm 14.3^{\mathrm{c}}$ & $532.4 \pm 3.4^{\mathrm{c}}$ & $450.8 \pm 13.2^{\mathrm{c}}$ \\
SCO + DZ & $322.1 \pm 23.5$ & $27.0 \pm 1.6^{* * *}$ & $201.3 \pm 19.4^{* * *}$ & $320.9 \pm 3.6^{* * *}$ & $1289.5 \pm 17.8^{* * *}$ \\
SCO + TA & $720.1 \pm 85.6^{*}$ & $40.5 \pm 7.4^{* * *}$ & $743.6 \pm 34.9^{* * *}$ & $409.2 \pm 2.2^{* * *}$ & $1176.2 \pm 34.4^{* * *}$ \\
SCO + E29 & $561.9 \pm 30.3^{*}$ & $16.3 \pm 1.3^{* * *}$ & $191.1 \pm 15.5^{* * *}$ & $332.8 \pm 2.2^{* * *}$ & $1139.9 \pm 39.3^{* * *}$ \\
SCO + E57 & $319.4 \pm 11.5$ & $17.9 \pm 1.1^{* * *}$ & $187.9 \pm 10.8^{* * *}$ & $331.2 \pm 3.8^{* * *}$ & $1352.3 \pm 26.4^{* * *}$ \\
SCO + 114 & $544.3 \pm 33.2^{* *}$ & $28.3 \pm 1.8^{* * *}$ & $260.4 \pm 13.2^{* * *}$ & $327.2 \pm 2.6^{* * *}$ & $1466.3 \pm 21.1^{* * *}$ \\
\hline
\end{tabular}

Each value represents the average $\pm \mathrm{SEM} ; n=7 .{ }^{\mathrm{c}} p<0.001 v s$. normal control. ${ }^{*} p<0.05 ;{ }^{* * *} p<0.001 v$ s. negative control; DW: distilled water $(10 \mathrm{ml} / \mathrm{kg}) ; \mathrm{SCO}$ : scopolamine (1 mg/kg); DZ: donepezil (1.2 mg/kg); TA: tacrine (10 mg/kg); E29, E57, and E114: aqueous extract of $Z$. jujuba at respective doses of 29, 57, and $114 \mathrm{mg} / \mathrm{kg}$. DW + DW: normal control group; SCO + DW: negative control group; SCO + DZ: positive control group treated with donepezil; SCO + TA: positive control group with tacrine; SCO + E29-E114: test groups treated with Z. jujuba extract; GSH: reduced glutathione; MDA: malondialdehyde; SOD: superoxide dismutase.

TAвLE 3: Effect of $Z$. jujuba extract on some oxidative stress markers in the prefrontal cortex.

\begin{tabular}{lccccc}
\hline \multirow{2}{*}{ Treatment } & & \multicolumn{3}{c}{ Oxidative stress markers in the prefrontal cortex } \\
& GSH $(\mu \mathrm{mol} / \mathrm{g})$ & MDA $(\mathrm{mmol} / \mathrm{g})$ & SOD $($ unit/min/g) & Nitrite $(\mathrm{mmol} / \mathrm{g})$ & Total protein $(\mu \mathrm{g} / \mathrm{ml})$ \\
\hline DW + DW & $1134.1 \pm 102.4$ & $4.5 \pm 0.5$ & $61.3 \pm 5.1$ & $169.6 \pm 1.7$ & $2170.9 \pm 36.1$ \\
SCO + DW & $232.6 \pm 33.7^{\mathrm{c}}$ & $13.3 \pm 2.3^{\mathrm{c}}$ & $212.6 \pm 373.2^{\mathrm{c}}$ & $613.6 \pm 2.8^{\mathrm{c}}$ & $331.6 \pm 32.2^{\mathrm{c}}$ \\
SCO + DZ & $487.3 \pm 49.2$ & $7.2 \pm 2.6^{*}$ & $126.5 \pm 16.6^{* * *}$ & $402.7 \pm 2.4^{* * *}$ & $1202.2 \pm 33.9^{* * *}$ \\
SCO + TA & $720.9 \pm 75.2^{* *}$ & $6.2 \pm 1.1^{* *}$ & $59.4 \pm 7.3^{* * *}$ & $530.4 \pm 3.2^{* * *}$ & $1404.1 \pm 25.1^{* * *}$ \\
SCO + E29 & $848.1 \pm 87.9^{* * *}$ & $9.5 \pm 0.7$ & $271.8 \pm 24.1^{* * *}$ & $370.4 \pm 3.5^{* * *}$ & $958.6 \pm 28.4^{* * *}$ \\
SCO + E57 & $710.4 \pm 79.9^{* *}$ & $9.5 \pm 1.2$ & $395.1 \pm 48.3^{* * *}$ & $366.4 \pm 2.8^{* * *}$ & $865.3 \pm 26.7^{* * *}$ \\
SCO + 114 & $538.1 \pm 56.9$ & $5.3 \pm 0.5^{* *}$ & $321.5 \pm 27.5^{* * *}$ & $425.2 \pm 1.1^{* * *}$ & $683.9 \pm 32.4^{* * *}$ \\
\hline
\end{tabular}

Each value represents the average \pm SEM; $n=7 .{ }^{c} p<0.001 v s$. normal control. ${ }^{*} p<0.05 ;{ }^{* *} p<0.01 ;{ }^{* * *} p<0.001 v s$. negative control; DW: distilled water $(10 \mathrm{ml} / \mathrm{kg})$; SCO: scopolamine $(1 \mathrm{mg} / \mathrm{kg}) ;$ DZ: donepezil $(1.2 \mathrm{mg} / \mathrm{kg}) ; \mathrm{TA}$ : tacrine $(10 \mathrm{mg} / \mathrm{kg}) ; \mathrm{E} 29, \mathrm{E} 57$, and E114: aqueous extract of $Z$. jujuba at respective doses of 29, 57, and $114 \mathrm{mg} / \mathrm{kg}$. DW + DW: normal control group; SCO + DW: negative control group; SCO + DZ: positive control group with donepezil; SCO + TA: positive control group treated with tacrine; SCO + E29-E114: test groups treated with Z. jujuba extract; GSH: reduced glutathione; MDA: malondialdehyde; SOD: superoxide dismutase.

activity (Tables 2 and 3 ) in both organs. In the hippocampus and prefrontal cortex, the concentration of nitrite significantly $(p<0.001)$ increased in the negative control group when compared to the normal control group (Tables 2 and 3). The extract at the doses of 29 and $114 \mathrm{mg} /$ $\mathrm{kg}$, donepezil, and tacrine showed the most remarkable $(p<0.001)$ decrease in the concentration of nitrite in both organs (Tables 2 and 3 ).

In the hippocampus and prefrontal cortex, administration of scopolamine caused a significant $(p<0.001)$ decrease in the concentration of total protein between the negative control group and the normal control group (Tables 2 and 3). However, the extract (29 and $144 \mathrm{mg} / \mathrm{kg}$ ), donepezil, and tacrine significantly $(p<0.001)$ increased this concentration in the two organs (Tables 2 and 3 ).

\subsection{Effect of Z. jujuba Extract on Some Proinflammatory} Markers in the Hippocampus and Prefrontal Cortex. The effect of the extract of $Z$. jujuba on some proinflammatory markers in the hippocampus and prefrontal cortex is shown in Figure 4. The negative control group showed a significant $(p<0.001)$ increase in the concentration of TNF- $\alpha$ in the hippocampus and prefrontal cortex $(158.4 \pm 1.3 \mathrm{pg} / \mathrm{ml}$ and $2148.9 \pm 1.7 \mathrm{pg} / \mathrm{ml}$, respectively) when compared to the normal control group (Figure 4(a)). The extract $(114 \mathrm{mg} / \mathrm{kg}$ ) showed the highest $(p<0.001)$ reduction of this concentration in the hippocampus and prefrontal cortex (62.47\% and $78.96 \%$, respectively) (Figure 4(a)). Similarly, donepezil and tacrine significantly $(p<0.001)$ decreased the TNF- $\alpha$ concentration in the hippocampus $(42.32 \%$ and $28.76 \%$, respectively) and prefrontal cortex (78.06\% and $73.16 \%$, respectively) (Figure 4(a)).The negative control group showed a significant $(p<0.001)$ increased IL-1 $\beta$ concentration in the hippocampus $(112.1 \pm 0.8 \mathrm{pg} / \mathrm{ml})$ and prefrontal cortex $(1281.3 \pm 1.1 \mathrm{pg} / \mathrm{ml})$ when compared to the normal control group (Figure 4(b)). The extract $(29 \mathrm{mg} / \mathrm{kg}$ ), donepezil, and tacrine significantly $(p<0.001)$ decreased the IL- $1 \beta$ concentration in the hippocampus and prefrontal cortex to $79.69 \%, 55.66 \%$, and $61.04 \%$, respectively (Figure 4(b)), while in the prefrontal cortex, the extract $(114 \mathrm{mg} / \mathrm{kg})$, donepezil, and tacrine significantly $(p<0.001)$ increased the IL- $1 \beta$ concentration to $60.12 \%, 36.04 \%$, and $45.42 \%$, respectively (Figure $4(\mathrm{~b})$ ). The administration of scopolamine in the negative control group significantly $(p<0.001)$ increased the concentration of IL- 6 in the hippocampus and prefrontal cortex to $346.1 \pm 8.7 \mathrm{pg} / \mathrm{ml}$ and $3526.3 \pm 3.4 \mathrm{pg} / \mathrm{ml}$, respectively, when compared to the negative control group (Figure 4(c)). The extract at all doses, as well as donepezil or tacrine, significantly $(p<0.001)$ decreased the concentration of IL- 6 in the hippocampus and prefrontal cortex (Figure 4(c)). 


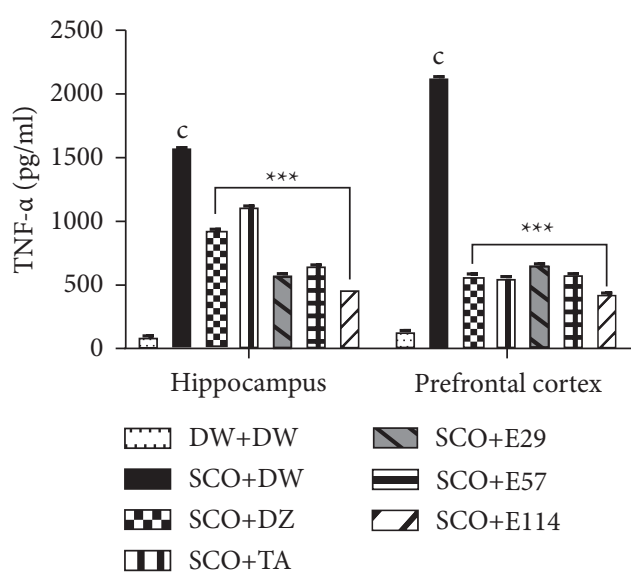

(a)

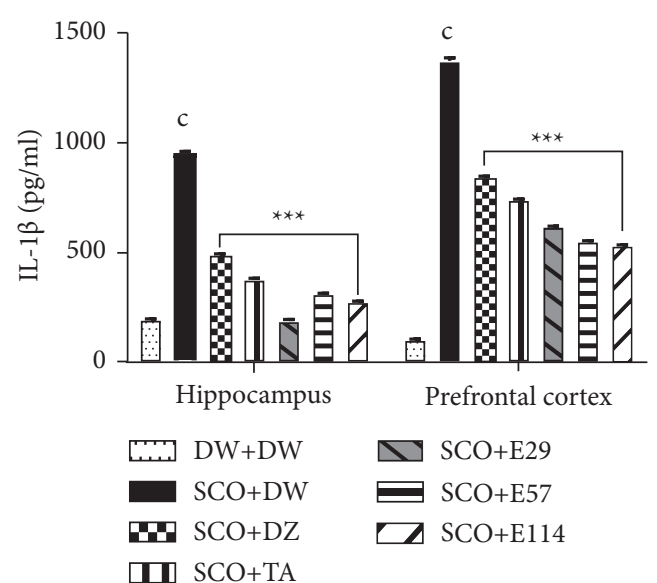

(b)

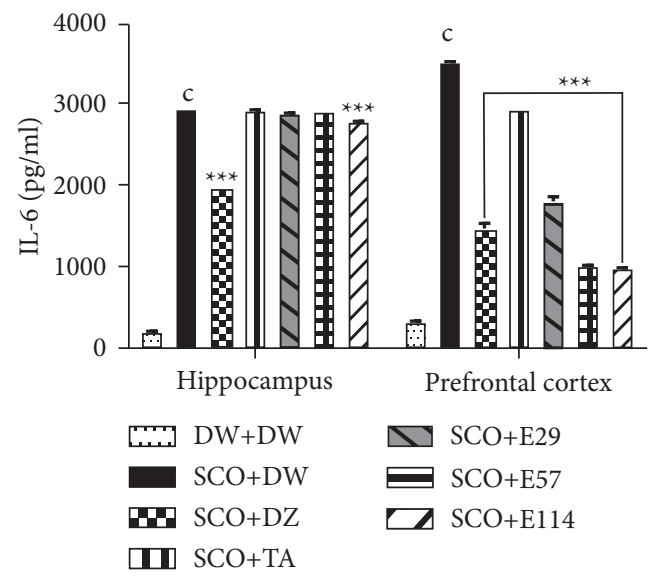

(c)

FIGURE 4: Effect of Z. jujuba extract on some proinflammatory markers in the hippocampus and prefrontal cortex. Each value represents the average \pm SEM; $n=7$. ${ }^{c} p<0.01$ vs. normal control. ${ }^{* * *} p<0.001$ vs. negative control. DW: distilled water $(10 \mathrm{ml} / \mathrm{kg})$; SCO: scopolamine $(1 \mathrm{mg} / \mathrm{kg}) ; \mathrm{DZ}$ : donepezil (1.2 mg/kg); TA: tacrine (10 mg/kg); and E29, E57, and E114: aqueous extract of Z. jujuba at respective doses of 29, 57 , and $114 \mathrm{mg} / \mathrm{kg}$. DW + DW: normal control group; SCO + DW: negative control group; SCO + DZ: positive control group treated with donepezil; SCO + TA: positive control group treated with tacrine; and SCO + E29-E114: test groups treated with $Z$. jujuba extract.

3.6. Effect of Z. jujuba Extract on Some Apoptosis Markers in the Hippocampus and Prefrontal Cortex. The effect of the extract Z. jujuba on some apoptosis markers is shown in Figure 5. In the negative control group, the concentration of caspase 3 significantly $(p<0.001)$ increased in the hippocampus and prefrontal cortex $(18.5 \pm 0.1 \mathrm{ng} / \mathrm{ml}$ and $25.4 \pm 0.1 \mathrm{ng} / \mathrm{ml}$, respectively) when compared to the normal control group (Figure 5(a)). In the hippocampus, the extract of $Z$. jujuba (144 mg/kg), donepezil, and tacrine significantly $(p<0.001)$ decreased this concentration to $87.94 \%, 39.28 \%$, and $24.03 \%$, respectively, while in the prefrontal cortex, the extract $(29 \mathrm{mg} / \mathrm{kg})$, donepezil, and tacrine significantly $(p<0.001)$ decreased this concentration to $60.95 \%, 66.23 \%$, and $59.05 \%$, respectively (Figure 5(a)). In the negative control group, the concentration of caspase 9 significantly $(p<0.001)$ increased in the hippocampus and prefrontal cortex $(84.7 \pm 0.1 \mathrm{ng} / \mathrm{ml}$ and $78.6 \pm 0.2 \mathrm{ng} / \mathrm{ml}$, respectively) when compared to the normal control group (Figure 5(b)). In contrast, the extract $(114 \mathrm{mg} / \mathrm{kg})$, donepezil, and tacrine significantly decreased $(p<0.001)$ the concentration of caspase 9 in the hippocampus $(64.67 \%, 59.09 \%$, and $53.43 \%$, respectively) and prefrontal cortex $(40.34 \%, 29.88 \%$, and $33.58 \%$, respectively) (Figure 5(b)).

3.7. Effect of Z. jujuba Extract on Neuronal Alterations in the Hippocampus and Prefrontal Cortex. The microarchitecture of the hippocampus of the normal control group showed intact neurons in CA1 and CA3 layers (Figure 6(A and B)) and regular thickness in the dentate gyrus, while in the prefrontal cortex, it was observed a normal density of neurons (Figure 6 (D)). Equally, the hippocampus of rats treated with the extract ( 29 and $57 \mathrm{mg} / \mathrm{kg}$ ), as well as donepezil, showed a regular thickness of CA1 and CA3 layers (Figure $6(\mathrm{Q}, R, \mathrm{U}$, and $\mathrm{V})$ ) and decreased perivascular edema (Figure $6(\mathrm{~S}$ and $\mathrm{W})$ ), while in the prefrontal cortex, it was observed a normal density of neurons (Figure 6 ( $\mathrm{T}$ and $\mathrm{X})$ ). Compared to the abovementioned treatments, the hippocampus of the negative control group showed a thickness of CA1 and CA3 layers, reduced density of neural 


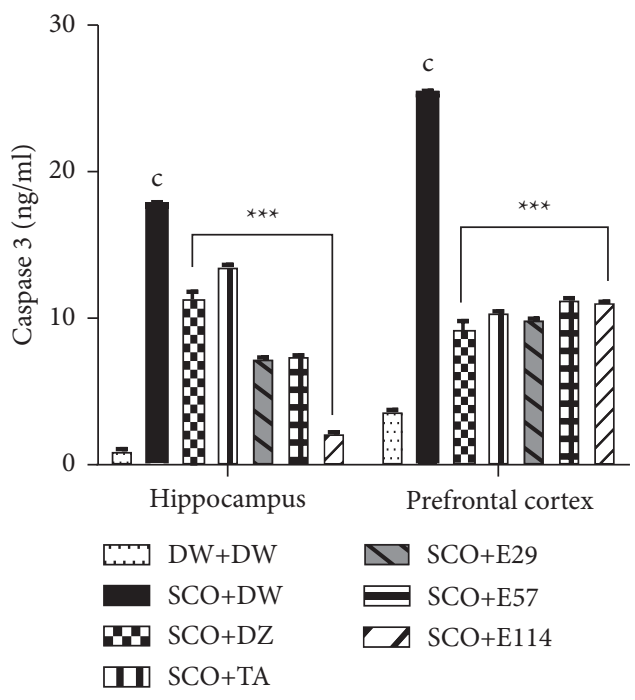

(a)

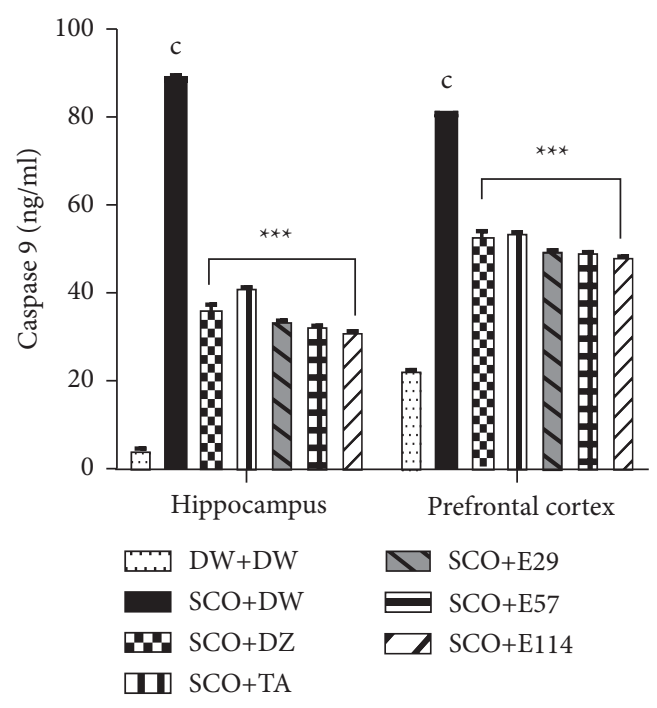

(b)

Figure 5: Effect of Z. jujuba extract on some apoptosis markers in the hippocampus and prefrontal cortex. Each value represents the average \pm SEM; $n=7$. ${ }^{c} p<0.01$ vs. normal control. ${ }^{* * *} p<0.001 v s$. negative control. DW: distilled water (10 ml $\left./ \mathrm{kg}\right)$; SCO: scopolamine $(1 \mathrm{mg} / \mathrm{kg}) ; \mathrm{DZ}$ : donepezil (1.2 mg/kg); TA: tacrine (10 mg/kg); and E29, E57, and E114: aqueous extract of $Z$. jujuba at respective doses of 29, 57 , and $114 \mathrm{mg} / \mathrm{kg}$. DW + DW: normal control group; SCO + DW: negative control group; SCO + DZ: positive control group treated with donepezil; SCO + TA: positive control group treated with tacrine; and SCO + E29-E114: test groups treated with $Z$. jujuba extract.

cell bodies, spongiosis (S), granulovacuolar degeneration (GVD), and chromatolysis (CH) (Figure 6 (E and 6F)); it was also observed the presence of perivascular edema (PE) in the dentate gyrus (Figure $6(\mathrm{G})$ ). Furthermore, the prefrontal cortex of this group showed a low density of neurons (Figure $6(\mathrm{H})$ ).

\subsection{Effect of Z. jujuba Extract on the Density of CA1 and CA3} Neurons in the Hippocampus. Table 4 presents the effect of the extract of $Z$. jujuba on the density of CA1 and CA3 neurons in the hippocampus. The negative control group showed a $45 \%(p<0.001)$ and $38 \%(p<0.05)$ decrease in the density of CA1 and CA3 neurons, respectively, as compared to the normal control group. Nonetheless, the extract (29 and $57 \mathrm{mg} / \mathrm{kg}$ ) and donepezil showed the highest $(p<0.05-0.001)$ increase in the density of CA1 and CA3 neurons, whereas tacrine only increased $(p<0.05)$ this density in the CA1 layer (Table 4).

3.9. Qualitative Phytochemical Analysis of Z. jujuba Extract. Qualitative phytochemical screening of the $Z$. jujuba aqueous extract indicated flavonoids, phenols, anthraquinones, coumarins, tannins, triterpenes, anthocyanins, phenols, and reducing sugars, but no significant alkaloids and saponins.

3.10. Quantitative Phytochemical Analysis of Z. jujuba Extract. Compared to standards, total phenolic compounds $(133.22 \pm 0.24 \mathrm{mg}$ gallic acid equivalent $/ \mathrm{g})$, condensed tannins $(20.66 \pm 0.21 \mathrm{mg}$ catechin equivalent/g), and flavonoid contents $\quad(11.99 \pm 0.68 \mathrm{mg}$ rutin equivalent/g) were abundant, while total alkaloids $(7.24 \pm 0.37 \%)$ and saponins $(3.66 \pm 0.23 \%)$ were less abundant.

\section{Discussion}

Alzheimer's disease (AD) is an irreversible, progressive brain disease that destroys memory and thinking skills and, eventually, the ability to perform the simplest tasks [21]. Until now, there is no curative treatment for AD. Thus, natural resources such as medicinal plants have been used to treat various memory disorders such as dementia, $\mathrm{AD}$, and Parkinson's disease for a long time [21]. Previous studies demonstrated that an aqueous macerate of $Z$. jujuba alleviates working memory impairment and restores neurochemical changes in the prefrontal cortex of D-galactosetreated rats [22]. However, the antiamnesic effect of an aqueous extract (concoction) of $Z$. jujuba was not assessed on learning and memory deficits. Therefore, this study investigated the effect of an aqueous extract (concoction) of $Z$. jujuba on scopolamine-induced cognitive impairments in rats using the Morris water maze and Y-maze paradigms. It is well known that memory impairments, especially those focused on episodic memory, are one of the major symptoms of $\mathrm{AD}$ [23], and the Morris water maze is commonly used to assess this type of memory impairment in rodents. The Morris water maze is a powerful and sensitive tool for evaluating hippocampal-spatial learning and reference memory. It is also used to identify drugs with antiamnesic properties, i.e., drugs preventing or restoring or reducing memory loss following brain injury [24]. In the present study, the administration of scopolamine for 15 days decreased the latency to find the platform during the acquisition phase (day 1 to day 4). However, on the 4th day of this 


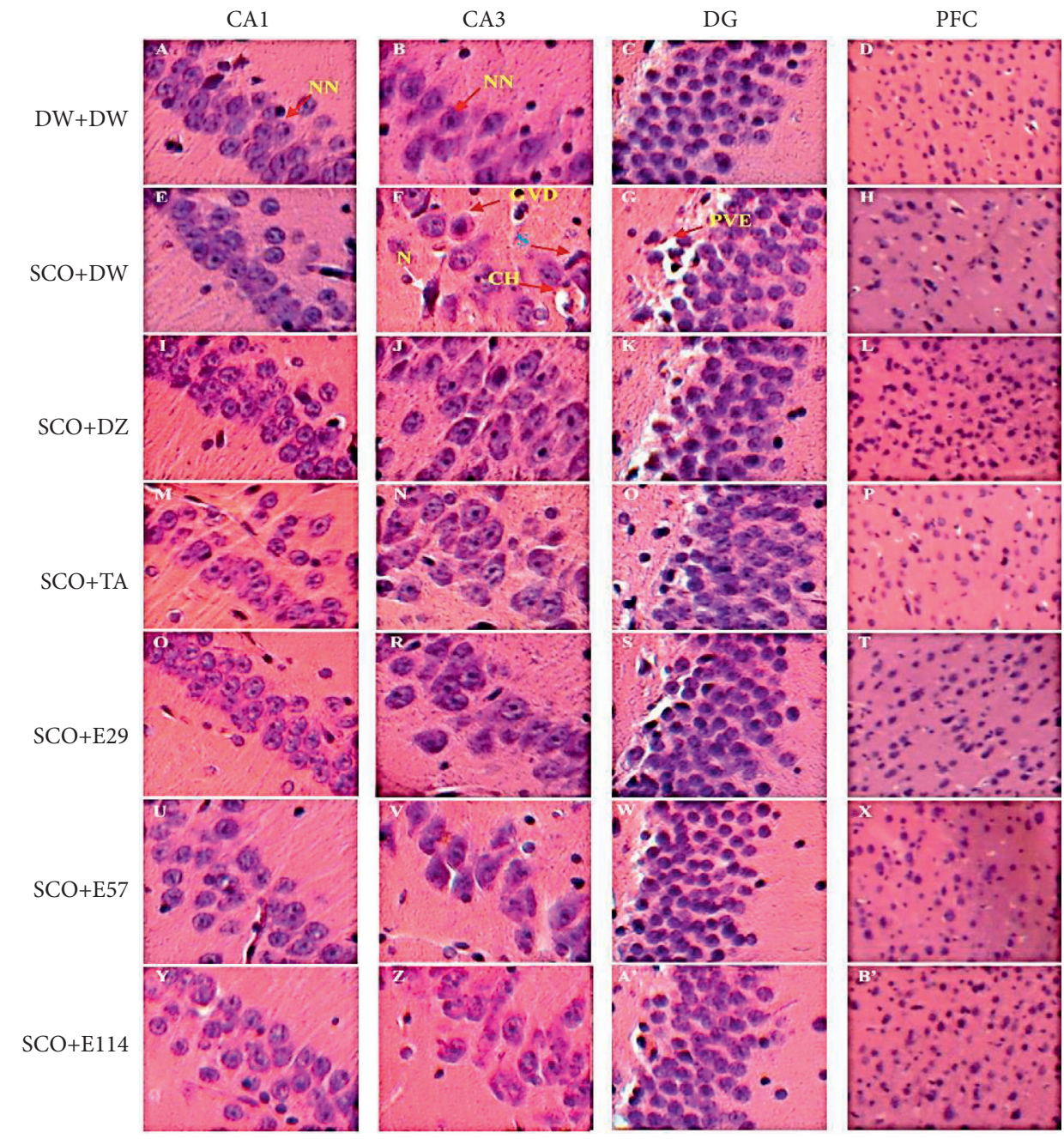

FIGURE 6: Photographs of the hippocampal and prefrontal cortex sections after hematoxylin and eosin staining (250X). Hippocampus (CA1, CA3, and DG); PFC: prefrontal cortex; CA1 and 3: Cornu ammonis 1 et 3; DG: dentate gyrus; N: neuron; NN: normal neuron; GVD: granulovacuolar degeneration; S spongiosis; CH: chromatolysis; PVE: perivascular edema; DW: distilled water (10 ml/kg); SCO: scopolamine $(1 \mathrm{mg} / \mathrm{kg}) ; \mathrm{DZ}$ : donepezil $(1.2 \mathrm{mg} / \mathrm{kg})$; TA: tacrine $(10 \mathrm{mg} / \mathrm{kg})$; and E29, E57, and E114: aqueous extract of $Z$. jujuba at respective doses of 29, 57, and $114 \mathrm{mg} / \mathrm{kg}$. DW + DW: normal control group; SCO + DW: negative control group; SCO + DZ: positive control group treated with donepezil; SCO + TA: positive control group treated with tacrine; and SCO + E29-E114: test groups treated with the extract of Z. jujuba.

TABLE 4: Effect of $Z$. jujuba extract on the density of CA1 and CA3 neurons in the hippocampus.

\begin{tabular}{lccccccc}
\hline & DW + DW & SCO + DW & SCO + DZ & SCO + TA & SCO + E29 & SCO + E57 & SCO + 114 \\
\hline CA1 & $110.1 \pm 3.1$ & $61.1 \pm 1.7^{\mathrm{c}}$ & $88.6 \pm 2.3^{* * *}$ & $74.1 \pm 1.1^{*}$ & $91.7 \pm 1.2^{* * *}$ & $85.3 \pm 3.7^{* * *}$ & $78.5 \pm 1.5^{* *}$ \\
CA3 & $46.3 \pm 1.5$ & $37.5 \pm 1.5^{\mathrm{a}}$ & $44.1 \pm 2.4^{*}$ & $41.5 \pm 1.5$ & $45.5 \pm 1.5^{*}$ & $45.1 \pm 1.1^{*}$ & $40.5 \pm 0.5$ \\
\hline
\end{tabular}

Each value represents the average $\pm \mathrm{SEM} ; n=7 .{ }^{\mathrm{a}} p<0.05 ;{ }^{\mathrm{c}} p<0.001$ vs. normal control. ${ }^{*} p<0.05 ;{ }^{* *} p<0.01 ;{ }^{* * *} p<0.001$ vs. negative control; DW: distilled water $(10 \mathrm{ml} / \mathrm{kg})$; SCO: scopolamine $(1 \mathrm{mg} / \mathrm{kg}) ; \mathrm{DZ}$ : donepezil $(1.2 \mathrm{mg} / \mathrm{kg})$; TA: tacrine $(10 \mathrm{mg} / \mathrm{kg}) ; \mathrm{E} 29$, E57, and E114: aqueous extract of $Z$. jujuba at respective doses of 29,57 , and $114 \mathrm{mg} / \mathrm{kg}$. DW + DW: normal control group; SCO + DW: negative control group; SCO + DZ: positive control group with donepezil; SCO + TA: positive control group with tacrine; SCO + E29-E114: test groups treated with the extract of Z. jujuba; CA1 : Cornus ammonis 1; CA3 : Cornus ammonis 3 .

phase, scopolamine markedly increased this time compared to the normal group, suggesting, thus, impairment of learning functions in rats. Twenty-four hours following the acquisition phase, i.e., during the retention phase (6th day of the experiment), administration of scopolamine increased the latency to find the platform and reduced the time spent in the target quadrant, as expected, suggesting an impairment of the reference memory. These findings corroborate those of Li et al. [25]. Scopolamine induces amnesia by acting as a nonselective agonist of muscarinic acetylcholine receptors of the M1 and M5 subtypes, acetylcholine being a critical neurotransmitter involved in long-term learning and memory processing [26], thus leading to recognition memory (place and identity memory) 
impairment. Therefore, alteration of the cholinergic system is followed by impairment of recognition memory during the acquisition and retention phases [27, 28]. In contrast, during both phases, the extract of $Z$. jujuba at all the doses and the positive control agent donepezil reversed these alterations, indicating that the extract has improved the remembrance of the platform position and, hence, better longterm spatial learning and memory functions in rats compared to the normal control group. These findings suggest an antiamnesic effect of the extract on long-term spatial learning and memory deficit $[25,29]$ and agree with those demonstrating that an ethanolic extract of $Z$. jujuba ameliorates cognitive impairments in rodents [25]. The fact that the extract restored learning and memory impairments similarly to donepezil, an inhibitor of acetylcholinesterase activity, suggests a blockade of cholinergic pathways by the extract as well, although the specific molecular components and mechanisms for the effects of the extract remain to be established. These results also indicate a beneficial effect of the extract on long-term learning and memory dysfunctions $[25,29]$. Furthermore, bioactive molecules belonging to the classes of flavonoids, alkaloids, and tannins have been shown to improve long-term memory in rodents [30-32]; some of these compounds (flavonoids and tannins) were identified as present in abundance in the extract, revealing that they might be responsible for the antiamnesic properties of the extract. Further studies will be required to isolate bioactive molecules in the extract and study their individual effects on long-term learning and memory impairments.

Working memory impairments are one of the earlier symptoms of $\mathrm{AD}$, for example, causing $\mathrm{AD}$ patients to forget the question that they just asked [33]. The prefrontal cortex is a key brain area for working memory, and injury to this brain area may induce deficits in working memory [34]. These patterns can be modeled in rodents by an intraperitoneal administration of scopolamine, which leads to the phosphorylation of tau proteins, the subsequent formation of beta-amyloid protein in the brain, and working memory deficits [34]. The Y-maze paradigm is usually preferred to assess working memory impairments in rodents [34], and it is based on the willingness of rodents to explore new environments [34]. Thus, normal animals prefer to explore a different arm of the maze than the one they visited on their previous entry. In rats receiving scopolamine and distilled water for 15 days, the percentage of spontaneous alternations in maze arm entry decreased, indicating that the animal has forgotten the arm it just visited. These observations suggested that, thus, impairment of working memory was comparable to those obtained in a previous study with the hydromethanolic extract of Ziziphus mucronata on scopolamine-induced amnesia in rats [35]. Surprisingly, the extract at all the doses and all standards did not significantly affect the percentage of spontaneous alternations, suggesting that they do not interfere with working memory dysfunctions. Therefore, they do not exert antiamnesic properties on short-term memory impairments $[7,36,37]$. These findings contrast with those obtained in recent studies with the aqueous macerate of $Z$. jujuba on the D-galactose model and reveal that the aqueous macerate is more effective than the concoction of the same plant [22]; maybe the aqueous macerate of $Z$. jujuba contains bioactive molecules that interfere with the process of short-term memory. Further studies on short-term memory impairments should be performed to confirm the obtained results in the present study.

In $\mathrm{AD}$, the deposition of beta-4-amyloid proteins in the brain induces the generation of reactive oxygen species (ROS) $[33,38]$, and the increase in the level of these molecules is associated with learning and memory impairments through the alteration of proteins, lipids, and DNA in neurons $[33,38]$. Besides, a previous study measured antioxidant enzyme activity and peroxide content in the brain tissue of patients with AD. The results revealed that GSH and SOD activities were significantly lower than those in healthy individuals, while MDA levels were markedly increased [39]. In the present study, administration of scopolamine for 15 days increased MDA, SOD, and nitrite levels and decreased those of GSH and total protein, indicating overproduction of free radicals (increased MDA and nitrite), inactivation of detoxification systems (GSH depletion), and consumption of antioxidants (increased SOD activity) in the hippocampus and prefrontal cortex. These results, therefore, suggest increased oxidative stress in the hippocampus and prefrontal cortex [39] and are consistent with those of Isola et al. [40] and Ghasemi et al. [41]. It is well established that oxidative stress is among the first and fundamental mechanisms of cell damage following the administration of scopolamine [3]. Indeed, during scopolamine-induced cognitive impairment, oxidative damage of lipids by lipid peroxidation results in the production of a wide variety of end products, including MDA, which is accepted as a reliable oxidative stress marker of lipid peroxidation in learning and memory deficits [42]. This process also includes the depletion of GSH, an intracellular thiol-based antioxidant present in reduced form, and has an essential role in the cellular defence cascade against oxidative injury; it acts as a cofactor for glutathione peroxidase and prevents the generation of hydroxyl free radicals [42]. All these changes disrupt the normal antioxidative defence ability of brain tissue. However, the administration of the extract at all doses and donepezil or tacrine markedly counteracted the scopolamine-induced increase in SOD, nitrite, and MDA levels compared to the normal control group and suggest either the inhibition of the lipid peroxidation or SOD overproduction stimulation. Moreover, the extract also reversed the depletion of GSH and increased total protein in both brain regions, suggesting direct scavenging of ROS or GSH production stimulation. These findings, therefore, reveal that the extract possesses significant and potentially valuable antioxidant activity, and this activity was more prominent than that of donepezil or tacrine with light antioxidant properties [9]. These results are consistent with several prior studies which demonstrated the antioxidant properties of $Z$. jujuba [43-45] and with those of Yoo et al. [46]. They showed that the neuroprotective effect of a methanolic extract of $Z$. jujuba against ischemic damage in the gerbil was due to its antioxidant properties. Furthermore, the presence of phenols, alkaloids, and flavonoids in the extract, evidenced in this study, may 
account for its antioxidant properties since several studies have shown that these compounds' cytoprotective and neuroprotective properties are strongly correlated to their antioxidant potential $[47,48]$. Taken together, these findings likely explain, at least in part, the antiamnesic and neuroprotective effects of the extract [46].

Reactive oxygen species-caused oxidative stress is considerably correlated with neuroinflammation, which exacerbates neurodegenerative disorders and contributes to the progression of AD [49]. Neuroinflammation is not generally seen as a cause of $\mathrm{AD}$, but rather as a result of $\mathrm{AD}$, in which microglia are overactivated, resulting in the increased production of proinflammatory cytokines TNF- $\alpha$, IL- $1 \beta$, and IL- 6 , some of the main drivers of neuroinflammation [49]. Similarly, deficiencies in anti-inflammatory mechanisms may contribute to neuroinflammation in AD [50]. A potential mechanism for the increase in these proinflammatory cytokines is through the inhibition of nuclear factor kappa-B (NFKB) [51], a known signal transduction mediator that is mainly involved in the control of the synthesis of proinflammatory cytokines in the cell [50]. Therefore, drugs with antiinflammatory activity could contribute to halting neuronal loss and improving the learning and memory deficits in patients with $\mathrm{AD}$ [51].

In the present study, repeated administration of scopolamine to distilled-water-treated rats induced a strong inflammatory response characterized by increased levels of proinflammatory cytokines (TNF- $\alpha$, IL-1 $\beta$, and IL-6) levels in the hippocampus and prefrontal cortex as compared to the normal group and suggested severe inflammation in these brain regions. Following intraperitoneal injection of scopolamine, an inflammatory response occurs mainly in the limbic system, which is characterized by the upregulation of proinflammatory cytokines and the activation of glial cells [52]. Activated glial cells and the overexpression of proinflammatory cytokines have been correlated with the injury of the hippocampus and often prefrontal cortex [52]. The aqueous extract and donepezil or tacrine mitigated the inflammatory processes induced by scopolamine in the hippocampus and prefrontal cortex, suggesting antiinflammatory properties abundantly reported in the literature [51]. These data corroborate those demonstrated by Kandeda et al. [22] with the aqueous macerate of the same plant on the D-galactose model in rats. The extract's antiinflammatory properties likely contribute to its antiamnesic effect, although further studies are needed to confirm this mechanism. The anti-inflammatory properties of the extract are also consistent with the neuroprotective effect, given that drugs with anti-inflammatory activities are known to reduce or prevent damage induced by inflammation in the brain [53]. Furthermore, the identified chemical compounds in the extract, including flavonoids, alkaloids, phenols, and tannins, might underlie this activity [15] since these compounds have been shown to possess anti-inflammatory activities in some experimental models of brain diseases [54]. This extract could have therapeutic value in treating learning and memory deficits and various brain diseases associated with neuroinflammation.
Compelling evidence indicates that oxidative stress and inflammation can trigger apoptosis mechanisms as part of $\mathrm{AD}$ pathogenesis [55]. Therefore, inhibiting these dysfunctions and the resultant apoptosis may reduce neuronal loss and cognitive impairments. In the present study, scopolamine increased the concentration of caspases 3 and 9, two significant contributors to apoptosis, in both the hippocampus and prefrontal cortex of the negative control group, similar to the results of Demirci et al. [56] with scopolamine-induced dementia in aged rats. Several studies established that scopolamine induces the formation of beta4-amyloid protein (BAP) in the hippocampus and prefrontal cortex [57], and BAP, via caspases 3, 8, and 9, leads to apoptosis of neuronal cells and contributes to the pathophysiology of $\mathrm{AD}$ [58]. In addition, this drug is also responsible for the initiation of apoptosis by promoting the overexpression of the proapoptotic protein, Bax, leading to cell death by increasing caspase activator release in the brain tissues $[59,60]$. In the present study, scopolamine increased the levels of caspases 3 and 9 in the hippocampus and prefrontal cortex, confirming, thus, the abovementioned findings. Treatment with the extract at all doses as well as donepezil or tacrine resulted in a significant decrease in the concentration of caspases assessed by ELISA in both the hippocampus and prefrontal cortex, suggesting an alteration of apoptotic mechanisms. These results also indicate that the extract is likely to exhibit antiapoptotic activity, contributing to its neuroprotective effect $[13,61]$. These properties might involve the action of some of the most abundant secondary metabolites, such as flavonoids and polyphenols, found in the extract, with antiapoptotic potential [59, 60]. Further studies will be required to identify the specific components of the extract that mediate its effects on caspase expression and the regulatory pathways involved.

In both humans and animals, the prefrontal cortex and hippocampus and their communication have been reported to play an essential role in the encoding and retrieval of diverse memory processes [62]. Consequently, damage in these areas is associated with severe cognitive impairments [62]. Analysis of the histological sections of the scopolamine-treated rats showed an alteration in the structure of CA1 and CA3 areas of the hippocampus of the negative control group. It was also observed the same patterns in the prefrontal cortex. These histological modifications are confirmed by a decrease in the number of neurons of CA1 and CA3. These findings suggest damage in both regions and ensure those obtained in previous studies [62]. However, the extract (29 and $57 \mathrm{mg} / \mathrm{kg}$ ) or donepezil alleviated structural damage in the hippocampus and prefrontal cortex. These modifications were confirmed by a significant increase in the number of neurons in CA1 and CA3 areas of the hippocampus.

Given that CA1, CA3, and prefrontal cortex regions are involved in the learning and memory process [63], these findings are consistent with the neuroprotective effect of the extract. This neuroprotective effect is likely to be mediated by the antioxidant, anti-inflammatory, and antiapoptotic activities we have demonstrated for the extract. Indeed, 
several studies reported that oxidative stress, inflammation, and apoptosis are all involved in neuronal alterations or death in the brain [56].

Taken together, this study here suggests that this extract or specific components derived from it could be used to prevent many of the long-term learning and memory dysfunctions and other neurodegenerative processes that occur in patients with $\mathrm{AD}$, epilepsy, and Parkinson's disease. Further studies on isolated molecules from this extract will help establish the exact components and mechanisms involved in the potentially clinically relevant antiamnesic and neuroprotective effects of this natural plant extract.

\section{Conclusions}

These studies document the antiamnesic and neuroprotective effects of $Z$. jujuba aqueous extract on the scopolamine model of $\mathrm{AD}$ in rats. Treatment with the aqueous extract protected animals from long-term learning and memory impairments. Analysis of possible modes of action suggests that these effects may be mediated, at least in part, by the antioxidant, anti-inflammatory, and antiapoptotic activities documented for the extract. These effects of the extract were better than those of donepezil, a standard drug against $\mathrm{AD}$. The antiamnesic and neuroprotective effects of the extract shown here support the long-time empirical use of this extract to treat dementia in Cameroonian folk medicine. Furthermore, this extract or its components could be used as an adjunct to treat diseases associated with longterm learning and memory impairments such as $\mathrm{AD}$ and related memory disorders.

\section{Data Availability}

The datasets used and/or analyzed during the current study are available from the corresponding author on simple request.

\section{Disclosure}

An earlier version of the manuscript has been presented as a preprint in Research Square.

\section{Conflicts of Interest}

There are no conflicts of interest.

\section{Authors' Contributions}

TD, AKK, and ED designed the study, AKK and ED performed all behavioral studies, accomplished data analysis, and drafted the manuscript, TD, JK, JPD, and SD critically revised the manuscript for important intellectual content, and SL, DN, and FN helped indirectly during the study. All authors have read and approved the final manuscript.

\section{Acknowledgments}

The authors are thankful to Professor Desiré Dzeufiet from the University of Yaoundé I (Cameroon) for his help in histopathology and the Laboratory of Animal Physiology (University of Yaoundé I, Cameroon) for providing apparatus. The authors are also grateful to Professor Myron Toews from the University of Nebraska (USA) for the manuscript English editing.

\section{References}

[1] S. Shabani and M. A. Mirshekar, "Diosmin is neuroprotective in a rat model of scopolamine-induced cognitive impairment," Biomedicine and Pharmacotherapy, vol. 108, pp. 1376-1383, 2018.

[2] H.-J. Lee, H.-L. Kim, D.-Y. Lee, D.-R. Lee, B.-K. Choi, and S.-H. Yang, "Scrophularia buergeriana extract (Brainon) improves scopolamine-induced neuronal impairment and cholinergic dysfunction in mice through CREB-BDNF signaling pathway," Biomolecules and Therapeutics, vol. 11, no. 9, p. 4286, 2021.

[3] N. E. Kouémou, G. S. Taiwe, F. C. O. Moto et al., "Nootropic and neuroprotective effects of dichrocephala integrifolia on scopolamine mouse model of alzheimer's disease," Frontiers in Pharmacology, vol. 8, p. 847, 2017.

[4] K. M. Frick, E. T. Stillner, and J. Berger-Sweeney, "Mice are not little rats: species differences in a one-day water maze task," Neuroreport, vol. 11, no. 16, pp. 3461-3465, 2000.

[5] S. Harandi, L. Golchin, M. Ansari, A. Moradi, M. Shabani, and V. Sheibani, "Antiamnesic effects of walnuts consumption on scopolamine-induced memory impairments in rats," Basic and Clinical Neuroscience, vol. 6, no. 2, pp. 91-99, 2015.

[6] L. A. Pattanashetti, A. D. Taranalli, V. Parvatrao, R. H. Malabade, and D. Kumar, "Evaluation of neuroprotective effect of quercetin with donepezil in scopolamineinduced amnesia in rats," Indian Journal of Pharmacology, vol. 49, no. 1, pp. 60-64, 2017.

[7] K. Van der Borght, R. Havekes, T. Bos, B. J. L. Eggen, and E. A. Van der Zee, "Exercise improves memory acquisition and retrieval in the Y-maze task: relationship with hippocampal neurogenesis," Behavioral Neuroscience, vol. 121, no. 2, pp. 324-334, 2007.

[8] S. H. Alawdi, E. S. El-Denshary, M. M. Safar, H. Eidi, M.-O. David, and M. A. Abdel-Wahhab, "Neuroprotective effect of nanodiamond in alzheimer's disease rat model: a pivotal role for modulating NF- $\kappa \mathrm{B}$ and STAT3 signaling," Molecular Neurobiology, vol. 54, no. 3, pp. 1906-1918, 2017.

[9] A. K. Kandeda, G. S. Taiwe, F. C. O. Moto et al., "Pergularia daemia aqueous extract ameliorates seizures and prevents oxidative stress in mice model of epilepsy," Asian Journal of Pharmaceutical and Health Sciences, vol. 7, no. 2, pp. 1664-1670, 2017.

[10] K. Wilbur, F. Bernheim, and O. Shapiro, "Determination of lipid peroxidation," Archives of Biochemistry and Biophysics, vol. 24, pp. 305-310, 1949.

[11] F. Misra and I. Fridovish, "Determination of the level of superoxide dismutase in whole blood," Yale University Press New Haven, vol. 101, no. 1972, p. 109, 1972.

[12] D. Tsikas, "Analysis of nitrite and nitrate in biological fluids by assays based on the Griess reaction: appraisal of the Griess reaction in the L-arginine/nitric oxide area of research," Journal of chromatography. B, Analytical technologies in the biomedical and life sciences, vol. 851, no. 1-2, pp. 51-70, 2007.

[13] A. K. Kandeda, G. S. Taiwe, F. C. O. Moto et al., "Antiepileptogenic and neuroprotective effects of Pergularia daemia on pilocarpine model of epilepsy," Frontiers in Pharmacology, vol. 8, p. 440, 2017. 
[14] D. A. M. Zaia, F. R. Marques, and C. T. B. V. Zaia, "Spectrophotometric determination of total proteins in blood plasma: a comparative study among dye-binding methods," Brazilian Archives of Biology and Technology, vol. 48, no. 3, pp. 385-388, 2005.

[15] O. O. Odebiyi and E. A. Sofowora, "Phytochemical screening of Nigerian medicinal plants II," Lloydia, vol. 41, no. 3, pp. 234-46, 1978.

[16] A. Harborne, Phytochemical Methods a Guide to Modern Techniques of Plant Analysisp. 278, 3rd edition, Springer Science \& Business Media, Berlin, Germany, 1998.

[17] T. Hatami, S. A. Emami, S. S. Miraghaee, and M. Mojarrab, "Total phenolic contents and antioxidant activities of different extracts and fractions from the aerial parts of Artemisia biennis Willd," Iranian Journal of Pharmaceutical Research: IJPR, vol. 13, no. 2, pp. 551-9, 2014.

[18] A. A. Dehpour, M. A. Ebrahimzadeh, N. S. Fazel, and N. S. Mohammad, "Antioxidant activity of the methanol extract of Ferula assafoetida and its essential oil composition," Grasas Y Aceites, vol. 60, no. 4, pp. 405-412, 2009.

[19] K. Ba, E. Tine, J. Destain, N. Cissé, and P. J. B. Thonart, "Étude comparative des composés phénoliques, du pouvoir antioxydant de différentes variétés de sorgho sénégalais et des enzymes amylolytiques de leur malt," Biotechnologie, Agronomie, Société et Environnement, vol. 14, no. 1, pp. 131-139, 2010.

[20] D. H. S. Gracelin, A. Britto, and B. J. R. Kumar, "Qualitative and quantitative analysis of phytochemicals in five Pteris species," International Journal of Pharmacy and Pharmaceutical Sciences, vol. 5, no. 1, pp. 105-107, 2013.

[21] A. Roy, "Role of medicinal plants against Alzheimer's disease," International Journal of Computational and Applied Mathematics, vol. 11, no. 4, pp. 205-208, 2018.

[22] A. K. Kandeda, D. Nguedia, E. R. Ayissi, J. Kouamouo, and T. Dimo, "Ziziphus jujuba (rhamnaceae) alleviates working memory impairment and restores neurochemical alterations in the prefrontal cortex of D-galactose-treated rats," EvidenceBased Complementary and Alternative Medicine, vol. 2021, Article ID 6610864, 15 pages, 2021.

[23] C. V. Vorhees and M. T. Williams, "Morris water maze: procedures for assessing spatial and related forms of learning and memory," Nature Protocols, vol. 1, no. 2, pp. 848-858, 2006.

[24] M. S. Kim, D. Y. Lee, J. Lee et al., “Terminalia chebula extract prevents scopolamine-induced amnesia via cholinergic modulation and anti-oxidative effects in mice," BMC Complementary and Alternative Medicine, vol. 18, no. 1, pp. 136-211, 2018.

[25] H. E. Lee, S. Y. Lee, J. S. Kim et al., "Ethanolic extract of the seed of Zizyphus jujuba var. spinosa ameliorates cognitive impairment induced by cholinergic blockade in mice," Biomolecules and Therapeutics, vol. 21, no. 4, pp. 299-306, 2013.

[26] I. Klinkenberg, A. Blokland, and B. Reviews, "The validity of scopolamine as a pharmacological model for cognitive impairment: a review of animal behavioral studies," Neuroscience and Biobehavioral Reviews, vol. 34, no. 8, pp. 1307-1350, 2010.

[27] C. J. Tinsley, N. S. Fontaine-Palmer, M. Vincent et al., "Differing time dependencies of object recognition memory impairments produced by nicotinic and muscarinic cholinergic antagonism in perirhinal cortex," Learning and Memory, vol. 18, no. 7, pp. 484-492, 2011.

[28] W. Wu, R. C. Saunders, M. Mishkin, and J. Turchi, "Differential effects of $\mathrm{m} 1$ and $\mathrm{m} 2$ receptor antagonists in perirhinal cortex on visual recognition memory in monkeys,"
Neurobiology of Learning and Memory, vol. 98, no. 1, pp. 41-46, 2012.

[29] S. Kar, S. P. Slowikowski, D. Westaway, and H. T. Mount, "Interactions between $\beta$-amyloid and central cholinergic neurons: implications for Alzheimer's disease," Journal of Psychiatry and Neuroscience, vol. 29, no. 6, 2004.

[30] N. Jivad and Z. Rabiei, "A review study on medicinal plants used in the treatment of learning and memory impairments," Asian Pacific Journal of Tropical Biomedicine, vol. 4, no. 10, pp. 780-789, 2014.

[31] M. Pahuja, J. Mehla, K. H. Reeta, S. Joshi, and Y. K. Gupta, "Hydroalcoholic extract of Zizyphus jujuba ameliorates seizures, oxidative stress, and cognitive impairment in experimental models of epilepsy in rats," Epilepsy and Behavior, vol. 21, no. 4, pp. 356-363, 2011.

[32] M. Taati, M. Alirezaei, M. H. Moshkatalsadat et al., "Protective effects of Ziziphus jujuba fruit extract against ethanol-induced hippocampal oxidative stress and spatial memory impairment in rats," Journal of Medicinal Plants Research, vol. 5, no. 6, pp. 915-921, 2011.

[33] Z. Sezgin and Y. Dincer, “Alzheimer's disease and epigenetic diet," Neurochemistry International, vol. 78, pp. 105-116, 2014.

[34] A.-K. Kraeuter, P. C. Guest, and Z. Sarnyai, "The Y-Maze for assessment of spatial working and reference memory in mice, methods in molecular biology," in Pre-Clinical Models, pp. 105-111, Springer, Berlin, Germany, 2019.

[35] H. S. Foyet, E. Keugong Wado, H. H. Ngatanko Abaissou, E. A. Assongalem, and O. K. Eyong, "Anticholinesterase and antioxidant potential of hydromethanolic extract of Ziziphus mucronata (rhamnaceae) leaves on scopolamine-induced memory and cognitive dysfunctions in mice," Evidence-based Complementary and Alternative Medicine, vol. 2019, 2019.

[36] M. Sarter, G. Bodewitz, and D. N. Stephens, "Attenuation of scopolamine-induced impairment of spontaneous alternation behaviour by antagonist but not inverse agonist and agonist $\beta$-carbolines," Psychopharmacology, vol. 94, no. 4, pp. 491-495, 1988.

[37] L. Walrave, M. Vinken, G. Albertini, D. De Bundel, L. Leybaert, and I. J. Smolders, "Inhibition of connexin43 hemichannels impairs spatial short-term memory without affecting spatial working memory," Frontiers in Cellular Neuroscience, vol. 10, p. 288, 2016.

[38] B. Rita Cardoso, V. Silva Bandeira, W. Jacob-Filho, and S. M. Franciscato Cozzolino, "Selenium status in elderly: relation to cognitive decline," Journal of Trace Elements in Medicine \& Biology, vol. 28, no. 4, pp. 422-426, 2014.

[39] D. L. Marcus, C. Thomas, C. Rodriguez et al., "Increased peroxidation and reduced antioxidant enzyme activity in Alzheimer's disease," Experimental Neurology, vol. 150, no. 1, pp. 40-44, 1998.

[40] I. O. Ishola, A. A. Jacinta, and O. O. Adeyemi, "Corticohippocampal memory enhancing activity of hesperetin on scopolamine-induced amnesia in mice: role of antioxidant defense system, cholinergic neurotransmission and expression of BDNF," Metabolic Brain Disease, vol. 34, no. 4, pp. 979-989, 2019.

[41] S. Ghasemi, M. Moradzadeh, M. Hosseini, F. Beheshti, and H. R. Sadeghnia, "Beneficial effects of Urtica dioica on scopolamine-induced memory impairment in rats: protection against acetylcholinesterase activity and neuronal oxidative damage," Drug and Chemical Toxicology, vol. 42, no. 2, pp. 167-175, 2019. 
[42] K. Rajashri, S. Mudhol, M. Serva Peddha, and B. B. Borse, "Neuroprotective effect of spice oleoresins on memory and cognitive impairment associated with scopolamine-induced alzheimer's disease in rats," ACS Omega, vol. 5, no. 48, pp. 30898-30905, 2020.

[43] M. Goyal, D. Sasmal, and B. Nagori, "Review on ethnomedicinal uses, pharmacological activity and phytochemical constituents of Ziziphus mauritiana (Z. Jujuba lam., non Mill)," Spatula DD, vol. 2, no. 2, pp. 107-116, 2012.

[44] R. Mahajan and M. Chopda, "Phyto-Pharmacology of Ziziphus jujuba Mill-A plant review," Pharmacognosy Reviews, vol. 3, no. 6, p. 320, 2009.

[45] W.-H. Peng, M.-T. Hsieh, Y.-S. Lee, Y.-C. Lin, and J. Liao, "Anxiolytic effect of seed of Ziziphus jujuba in mouse models of anxiety," Journal of Ethnopharmacology, vol. 72, no. 3, pp. 435-441, 2000.

[46] K.-Y. Yoo, H. Li, I. K. Hwang et al., “Zizyphus attenuates ischemic damage in the gerbil hippocampus via its antioxidant effect," Journal of Medicinal Food, vol. 13, no. 3, pp. 557-563, 2010.

[47] H. P. Misra and I. Fridovich, "The role of superoxide anion in the autoxidation of epinephrine and a simple assay for superoxide dismutase," Journal of Biological Chemistry, vol. 247, no. 10 , pp. 3170-3175, 1972.

[48] B. D. Obay, E. Taşdemir, C. Tümer, H. M. Bilgin, and M. Atmaca, "Dose dependent effects of ghrelin on pentylenetetrazole-induced oxidative stress in a rat seizure model," Peptides, vol. 29, no. 3, pp. 448-455, 2008.

[49] E. E. Tuppo and H. R. Arias, "The role of inflammation in Alzheimer's disease," The International Journal of Biochemistry and Cell Biology, vol. 37, no. 2, pp. 289-305, 2005.

[50] S. Viau, B. Pasquis, M.-A. Maire et al., "No consequences of dietary n-3 polyunsaturated fatty acid deficiency on the severity of scopolamine-induced dry eye," Graefes Archive for Clinical and Experimental Ophthalmology, vol. 249, no. 4, pp. 547-557, 2011.

[51] P. L. McGeer, J. Rogers, and E. G. McGeer, "Inflammation, antiinflammatory agents, and alzheimer's disease: the last 22 years," Journal of Alzheimer's Disease, vol. 54, no. 3, pp. 853-857, 2016.

[52] S. Iqbal, F. A. Shah, K. Naeem et al., "Succinamide derivatives ameliorate neuroinflammation and oxidative stress in scopolamine-induced neurodegeneration," Biomolecules, vol. 10, no. 3, p. 443, 2020.

[53] P. Rathee, H. Chaudhary, S. Rathee, D. Rathee, V. Kumar, and K. Kohli, "Mechanism of action of flavonoids as anti-inflammatory agents: a review," Inflammation and Allergy Drug Targets, vol. 8, no. 3, pp. 229-235, 2009.

[54] S. M. Al-Reza, J. I. Yoon, H. J. Kim, J.-S. Kim, and S. C. Kang, "Anti-inflammatory activity of seed essential oil from Zizyphus jujuba," Food and Chemical Toxicology, vol. 48, no. 2, pp. 639-643, 2010.

[55] S. H. Kwon, H. K. Lee, J. A. Kim et al., "Neuroprotective effects of chlorogenic acid on scopolamine-induced amnesia via antiacetylcholinesterase and anti-oxidative activities in mice," European Journal of Pharmacology, vol. 649, no. 1-3, pp. 210-217, 2010.

[56] K. Demirci, M. Nazıroğlu, İ. S. Övey, and H. Balaban, "Selenium attenuates apoptosis, inflammation and oxidative stress in the blood and brain of aged rats with scopolamineinduced dementia," Metabolic Brain Disease, vol. 32, no. 2, pp. 321-329, 2017.

[57] W. Liskowsky and R. Schliebs, "Muscarinic acetylcholine receptor inhibition in transgenic Alzheimer-like Tg2576 mice by scopolamine favours the amyloidogenic route of processing of amyloid precursor protein," International Journal of Developmental Neuroscience, vol. 24, no. 2-3, pp. 149-156, 2006.

[58] M. Medina, J. J. Garrido, and F. G. Wandosell, "Modulation of GSK-3 as a therapeutic strategy on tau pathologies," Frontiers in Molecular Neuroscience, vol. 4, p. 24, 2011.

[59] J.-M. Chow, S.-C. Shen, S. K. Huan, H.-Y. Lin, and Y.-C. Chen, "Quercetin, but not rutin and quercitrin, prevention of $\mathrm{H} 2 \mathrm{O} 2$-induced apoptosis via anti-oxidant activity and heme oxygenase 1 gene expression in macrophages," Biochemical Pharmacology, vol. 69, no. 12, pp. 1839-1851, 2005.

[60] J.-i. Oyama, T. Maeda, M. Sasaki et al., "Green tea catechins improve human forearm vascular function and have potent anti-inflammatory and anti-apoptotic effects in smokers," Internal Medicine, vol. 49, no. 23, pp. 2553-2559, 2010.

[61] X. Wang, "The antiapoptotic activity of melatonin in neurodegenerative diseases," CNS Neuroscience and Therapeutics, vol. 15, no. 4, pp. 345-357, 2009.

[62] A. R. Preston and H. Eichenbaum, "Interplay of hippocampus and prefrontal cortex in memory," Current Biology, vol. 23, no. 17, pp. R764-R773, 2013.

[63] S. Daumas, H. Halley, B. Frances, and J. M. Lassalle, "Encoding, consolidation, and retrieval of contextual memory: differential involvement of dorsal CA3 and CA1 hippocampal subregions," Learning and Memory, vol. 12, no. 4, pp. 375-382, 2005. 